\title{
Diseño experimental de una solución polimérica para mejorar la relación de movilidad de los fluidos en un yacimiento previo a la implementación de un proyecto piloto de recuperación mejorada de petróleo
}

\section{(Experimental Design of a Polymeric Solution to Improve the Mobility Ratio in a Reservoir previous implementation of a pilot project of EOR)}

\author{
Vanessa Cuenca ${ }^{1}$, Rubén Paredes² ${ }^{2}$ José Cóndor ${ }^{3,1}$
}

\begin{abstract}
Resumen:
Este paper describe los trabajos realizados para formular experimentalmente soluciones poliméricas con el objetivo de determinar la concentración óptima para controlar la movilidad de los fluidos en un yacimiento previo a la implementación de un proyecto piloto de recuperación mejorada de petróleo. Los polímeros, en primer lugar, fueron seleccionados a base de las propiedades de fluidos obtenidos de un reservorio. La denominación de los polímeros fueron TCC-330 y EOR909 y las pruebas experimentales que se realizaron fueron: estabilidad térmica, compatibilidad, adsorción, salinidad, y desplazamiento. El diseño que mejores resultados mostró fue el del polímero TCC-330 a una concentración de 1.500 ppm.
\end{abstract}

Palabras clave: inyección de polímeros; recuperación mejorada de petróleo; relación de movilidad; ensayos de laboratorio.

\begin{abstract}
:
This paper describes experimental formulations of polymeric solutions through lab evaluations with the objective of finding optimum solution concentration to fluid mobility in reservoirs as previous step before implementing a pilot project of enhanced oil recovery. The polymers, firstly, were selected based on the properties from fluids from reservoir. Two types of polymers were used TCC-330 and EOR909 and the experimental tests were: thermal stability, compatibility, adsorption, salinity, and displacement. The design with the best results was with polymer TCC330 at $1,500 \mathrm{ppm}$ concentration.
\end{abstract}

Keywords: polymer injection; enhanced oil recovery; mobility ratio; laboratory test.

\footnotetext{
${ }^{1}$ Universidad Central del Ecuador, Quito - Ecuador (vanelucuenca@gmail.com)

2 Qmax, Quito - Ecuador (Rubens_Paredes@hotmail.com)

${ }^{3}$ Universidad Tecnológica Equinoccial, Quito - Ecuador (josea.condor@ute.edu.ec)
} 


\section{Introducción}

Es común clasificar la vida productiva de un reservorio en tres etapas. La primera es la producción a partir de la energía propia del reservorio. La etapa secundaria ocurre cuando la presión desciende y mediante la inyección de agua o gas se mantiene la presión del reservorio. El desplazamiento de petróleo en esta etapa es del tipo físico y mecánico (Stosur, Hite, Carnahan, \& Miller, 2003).

En las últimas décadas se han desarrollado tecnologías o métodos para producir el petróleo que no fue producido en las etapas primaria y secundaria. Estos métodos son conocidos como: Recuperación Terciaria o Mejorada de Petróleo (EOR por sus siglas en inglés). Se estima que el EOR puede recuperar entre 10 y 20 por ciento del petróleo original en sitio (Sánchez Barrios, 2013).

La recuperación mejorada de petróleo es el conjunto de técnicas que inyecta energía adicional para producir desplazamiento diferente al físico en el reservorio. Una de las técnicas EOR consiste en el uso de químicos tales como los polímeros y surfactantes. Dado que no es práctico cambiar las propiedades del petróleo o la permeabilidad de la roca, la mayoría de los procesos de control de movilidad buscan adicionar químicos a los fluidos inyectados. (Xu, Kim, \& Delshad, 2012)

La inyección de polímeros se introdujo con la finalidad de lograr el control y alterar la relación de movilidad. El método consiste en formar una mezcla de agua con moléculas químicas de polímero, las cuales crean una red (repetición de unidades químicas simples llamadas monómeros) que crean un material resistente y de alto peso molecular adecuado para el control de la producción de agua y recuperación de petróleo. El objetivo de esta técnica es aprovechar las viscosidades de las soluciones de polímeros en el control de la movilidad de los fluidos en el yacimiento (Carcoana, 1992; Salager, 2005).

El presente proyecto describe el desarrollo experimental de soluciones poliméricas para encontrar la concentración óptima que mejore la relación de movilidad en el yacimiento.

El trabajo de laboratorio tuvo como objetivo determinar y evaluar las propiedades físicas de las soluciones de polímeros formuladas. La comprensión de estas propiedades ayudará al diseño de un proceso de recuperación de petróleo con aplicación de polímeros. Se seleccionaron los polímeros de acuerdo con sus características de anionicidad, peso molecular y poder viscosificante, en soluciones de 500 a 1.500 ppm, para estudiar su comportamiento reológico a distintas concentraciones en función a la temperatura, se obtienen las respectivas curvas que posteriormente fueron linealizadas mediante la ecuación de Arrehenius. Luego se ejecutaron pruebas de salinidad, compatibilidad y adsorción, para evaluar su eficiencia en el proceso. Además, se diseñó un equipo para realizar pruebas de desplazamiento. Esto ayudó a evaluar 
visualmente el polímero de mejor desempeño para movilizar el crudo mediante núcleos de cerámica.

\section{Metodología}

Este estudio fue de tipo experimental apoyado por una investigación bibliográfica y de laboratorio (Barnes, 1962). El tema se lo desarrolló de manera íntegra en las instalaciones del Laboratorio de la Empresa Qmax Solutions Ecuador S.A. Este proyecto comprendió la descripción, formulación, análisis, e interpretación de datos de la inyección de polímeros para mejorar la movilidad de los fluidos en el yacimiento (Cuenca \& Cóndor, 2016). La Figura 1 detalla las etapas comprendidas de desarrollo del proyecto.
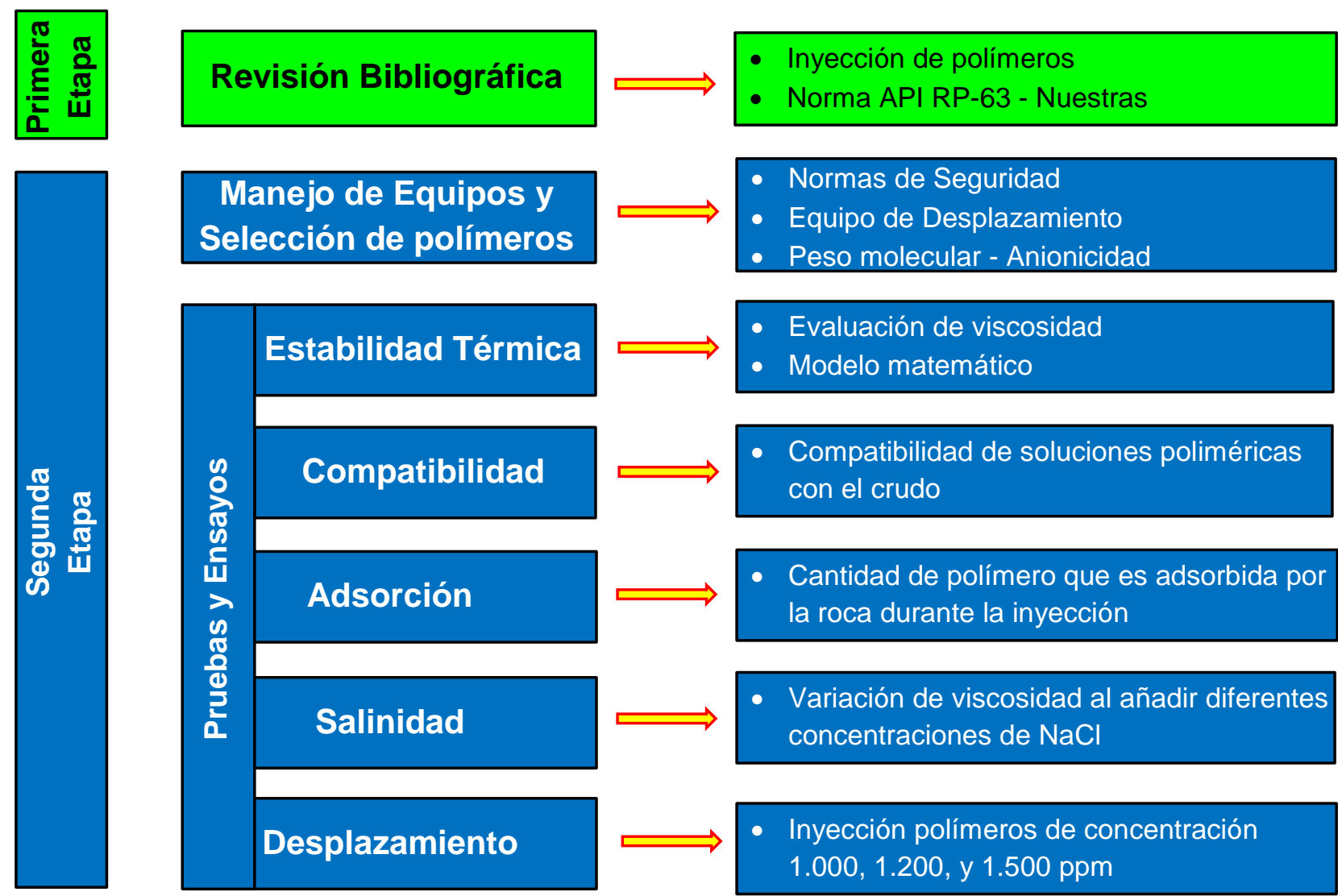

- Evaluación de viscosidad

- Modelo matemático

- Compatibilidad de soluciones poliméricas con el crudo

- Cantidad de polímero que es adsorbida por la roca durante la inyección

- Variación de viscosidad al añadir diferentes concentraciones de $\mathrm{NaCl}$

- Inyección polímeros de concentración $1.000,1.200$, y 1.500 ppm

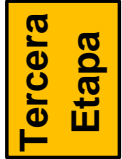

\section{Resultados y Análisis}

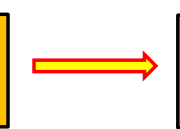

\section{- Conclusiones \\ - Recomendaciones}

Figura 1. Esquema de la metodología

\subsection{Primera Etapa}

Consistió en la revisión bibliográfica de artículos en revistas indexadas y libros acerca de:

- Antecedentes y estudios de inyección de químicos (Aktas, Clemens, Castanier, \& Kovscek, 2008; Abrahamsen, 2012; Buchgraber, 2008; Chinenye, 2010)

- Información técnica proporcionada por Qmax (Qmax Solutions S.A., 2015)

- Revisión de la Norma API RP-63 acerca de prácticas recomendadas para la evaluación de polímeros usadas en operaciones de Recuperación Mejorada de Petróleo (API, 1990). 
- Obtención de muestras del yacimiento "X" de crudo, agua de inyección y polímeros

\subsection{Segunda Etapa}

Pruebas de laboratorio para encontrar la concentración óptima de formulación polimérica. Estas pruebas se realizaron siguiendo la Norma API RP-63 para ensayos de estabilidad térmica, reología, compatibilidad, salinidad, adsorción y pruebas de desplazamiento.

\subsection{Tercera Etapa}

Los resultados obtenidos de las pruebas de laboratorio fueron resumidos mediante tablas y gráficos en hojas electrónicas Microsoft Excel. También se utilizó Geogebra, para la evaluación de las curvas de polímeros.

\section{Resultados y Discusión}

Con las muestras de campo obtenidas se realizaron las pruebas de laboratorio, y los resultados luego de ser evaluados, permitieron determinar el polímero candidato para ser inyectado en el área de estudio.

\subsection{Parámetros iniciales del pozo}

Para la preparación de las soluciones poliméricas y pruebas de desplazamiento se tomaron muestras de crudo y agua de inyección proporcionadas por la empresa operadora. Los parámetros iniciales más relevantes se resumen en la Tabla 1.

Tabla 1. Parámetros iniciales de crudo y agua de inyección del yacimiento "X
\begin{tabular}{cc}
\hline Parámetros & Agua de Inyección \\
\hline $\mathrm{pH}$ & 9.7 \\
\hline Conductividad & $27.8 \mathrm{mS} / \mathrm{cm}$ \\
\hline Sólidos disueltos totales (TDS) & $13.91 \mathrm{~g} / \mathrm{l} @ 21.9^{\circ} \mathrm{C}$ \\
\hline Turbidez & $0.34 \mathrm{NTU}$ \\
\hline Cloruros & $120 \mathrm{mg} / \mathrm{l}$ \\
\hline Parámetros & Crudo \\
\hline Gravedad específica & 0.96 @ $13^{\circ} \mathrm{C}$ \\
\hline API & $15.9 @ 13^{\circ} \mathrm{C}$ \\
\hline Viscosidad & $187.3 \mathrm{cP}$ \\
\hline Temperatura & $120^{\circ} \mathrm{F}$ \\
\hline
\end{tabular}

\subsection{Selección de los polímeros candidatos}

La revisión bibliográfica recomendó el uso de polímeros con las siguientes características (Demin, y otros, 1996; Pancharoen, 2009):

- Poliacrilamidas parcialmente hidrolizadas debido a su alta solubilidad en agua. Esto permite que se requieran tiempos de agitación bajos como punto inicial

- Polímeros de peso molecular alto para obtener una alta viscosidad que soporten temperaturas mayores a $160^{\circ} \mathrm{F}$ y de baja susceptibilidad a la salinidad

- Polímeros aniónicos debido a que los núcleos de cerámica estuvieron compuestos por moléculas de cuarzo y al interactuar con estos en la solución se puede crear un efecto de 
repulsión, el cual reduce la adsorción del polímero en el medio poroso y garantizando las interacciones intermoleculares entre el solvente (agua) y el polímero

Con base a estas características se decidió emplear los polímeros TCC-330 y EOR909 pues cumplieron con la mayoría de los parámetros (Tabla 2).

Tabla 2. Datos técnicos de los polímeros candidatos a emplearse (Qmax Solutions S.A., 2015)

\begin{tabular}{ccccc}
\hline Polímero & TCC-330 & EOR 909 & 130 B & 934 S \\
\hline Clase química & Poliacrilamida & Poliacrilamida & Poliacrilamida & Poliacrilamida \\
\hline Anionicidad & Alta & Alta & Media & Media Alta \\
\hline Peso molecular (millón) & $30+/-5 \%$ & $25.5+/-5 \%$ & $12+/-5 \%$ & $20+/-5 \%$ \\
\hline Viscosidad Aparente (mPa.s) & $>16$ & 12 & 5.26 & 10.1 \\
\hline
\end{tabular}

\subsection{Estudios de Estabilidad Térmica}

El ensayo de estabilidad térmica consiste en poder conocer el efecto de la temperatura y en la pérdida de viscosidad de las soluciones con diferentes concentraciones a diferentes temperaturas.

\section{a) Medición de la viscosidad de las soluciones de polímero}

Una propiedad muy importante de los polímeros es la habilidad para incrementar la viscosidad de la solución. Se tomaron los valores a una tasa de corte de 100 rpm de las soluciones de polímero a concentraciones de 500 a 1.500 ppm. La primera medición se realizó a temperatura ambiente, para luego calentarlos a temperaturas de 120, 140, 160, 180, y $220^{\circ} \mathrm{F}$ (Tabla 3). En la tabla se puede deducir que a temperaturas elevadas la viscosidad decrece, especialmente en soluciones con menor concentración de polímero como se muestra en la Figura 2.

Tabla 3: Viscosidad del agua en función de la temperatura a diferentes concentraciones de los polímeros TCC-330 y EOR909

\begin{tabular}{|c|c|c|c|c|c|c|c|c|c|}
\hline Producto & $\begin{array}{c}\text { Concentración } \\
(\mathrm{ppm})\end{array}$ & $\begin{array}{c}\mathrm{T} \\
\left({ }^{\circ} \mathrm{F}\right)\end{array}$ & $\begin{array}{c}\mu \\
(\mathrm{cP})\end{array}$ & $\begin{array}{c}\mathrm{T} \\
\left({ }^{\circ} \mathrm{F}\right)\end{array}$ & $\begin{array}{c}\mu \\
(\mathrm{cP})\end{array}$ & $\begin{array}{c}\mathrm{T} \\
\left({ }^{\circ} \mathrm{F}\right)\end{array}$ & $\begin{array}{c}\mu \\
(\mathrm{cP})\end{array}$ & $\begin{array}{c}\mathrm{T} \\
\left({ }^{\circ} \mathrm{F}\right)\end{array}$ & $\begin{array}{c}\mu \\
(\mathrm{cP})\end{array}$ \\
\hline TCC-330 & 500 & 72,8 & 148,8 & 120 & 86,4 & 140 & 62,4 & 160 & 38,3 \\
\hline TCC-330 & 1.000 & 78 & 250 & 120 & 187,2 & 140 & 162 & 160 & 128,7 \\
\hline TCC-330 & 1.200 & 79,1 & 315.3 & 120 & 230,4 & 140 & 206,4 & 160 & 171.5 \\
\hline TCC-330 & 1.500 & 75,4 & 355.1 & 120 & 278 & 140 & 247 & 160 & 211,3 \\
\hline Producto & $\begin{array}{c}\text { Concentración } \\
(\text { ppm) }\end{array}$ & $\begin{array}{c}\mathrm{T} \\
\left({ }^{\circ} \mathrm{F}\right)\end{array}$ & $\begin{array}{c}\mu \\
(\mathrm{cP})\end{array}$ & $\begin{array}{c}\mathrm{T} \\
\left({ }^{\circ} \mathrm{F}\right)\end{array}$ & $\begin{array}{c}\mu \\
(\mathrm{cP})\end{array}$ & $\begin{array}{c}\mathrm{T} \\
\left({ }^{\circ} \mathrm{F}\right)\end{array}$ & $\begin{array}{c}\mu \\
(\mathrm{cP})\end{array}$ & $\begin{array}{c}\mathrm{T} \\
\left({ }^{\circ} \mathrm{F}\right)\end{array}$ & $\begin{array}{c}\mu \\
(\mathrm{cP})\end{array}$ \\
\hline EOR909 & 500 & 72,8 & 48 & 120 & 33,6 & 140 & 29,6 & 160 & 17,5 \\
\hline EOR909 & 1.000 & 78 & 96 & 120 & 82 & 140 & 71,6 & 160 & 57,3 \\
\hline EOR909 & 1.200 & 79,1 & 115,2 & 120 & 92 & 140 & 82,2 & 160 & 71,3 \\
\hline EOR909 & 1.500 & 75,4 & 124.8 & 120 & 105 & 140 & 100,5 & 160 & 96 \\
\hline
\end{tabular}

La temperatura máxima a la cual se pudo determinar la viscosidad fue a $160^{\circ} \mathrm{F}$. No fue posible medir este valor a la temperatura de 180 y 220 ํ F debido a que se observó ebullición y formación de burbujas que generaron errores en las lecturas. Para ello se construyó un perfil de viscosidad en función de la temperatura para luego extrapolarlos a $220^{\circ} \mathrm{F}$. Se usó la ecuación linealizada de Arrehenius que es la expresión matemática que más se ajustó a las variables de diseño. Esta 
ecuación es usada como modelo de regresión lineal entre variables para corroborar la dependencia de la constante de velocidad de una reacción química con respecto a la temperatura (figuras 3 y 4 ).
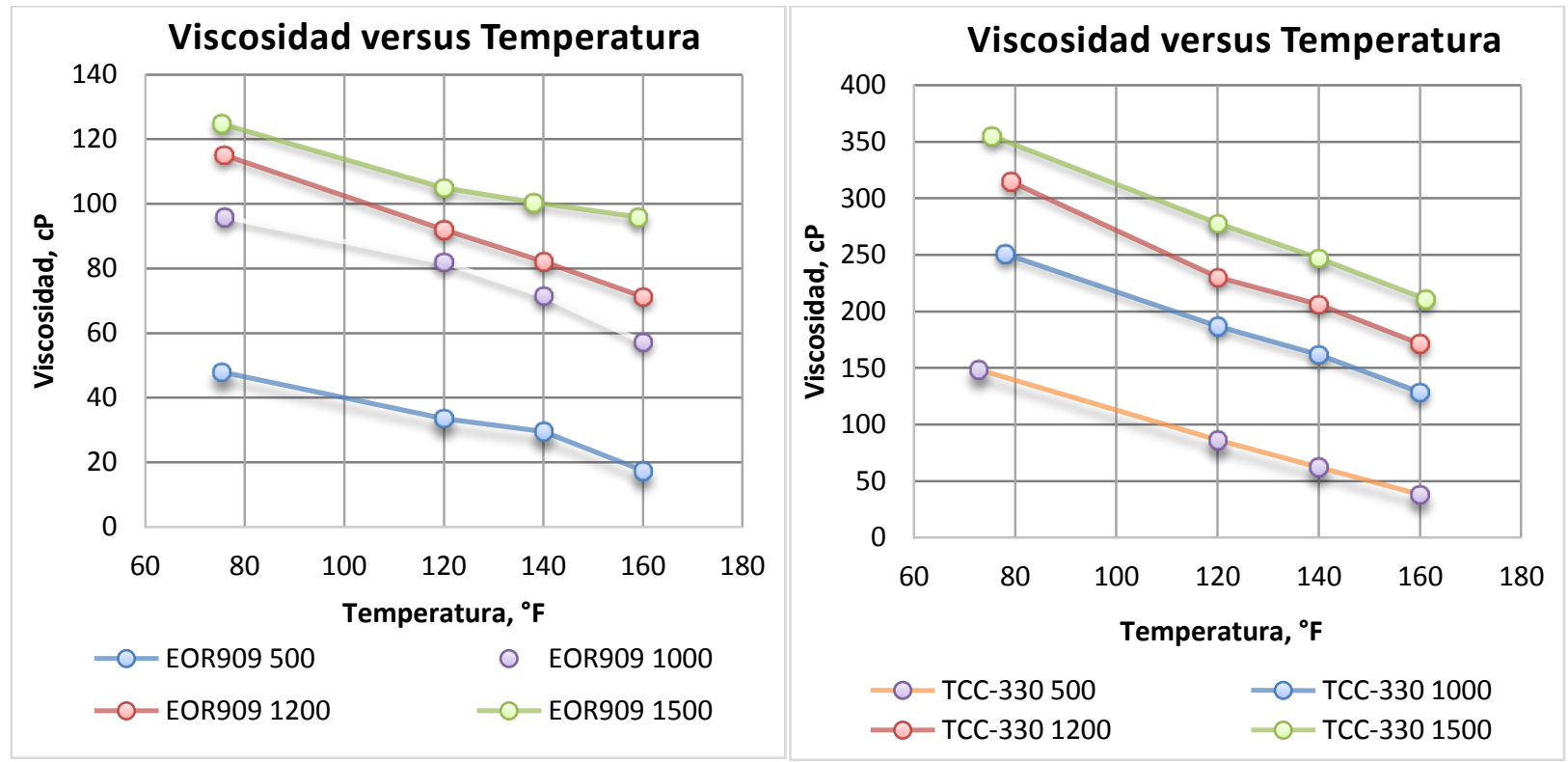

Figura 2. Viscosidad de la solución de agua en función de la temperatura

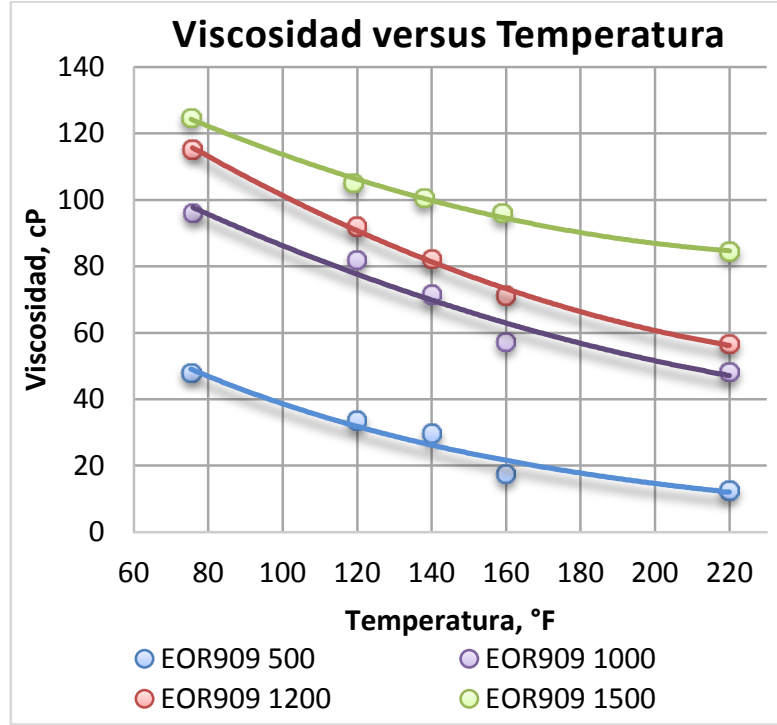

Figura 3. Resultados de Viscosidad de la solución de agua versus Concentración extrapolado a $220^{\circ} \mathrm{F}$ - EOR909

\section{b) Modelo Matemático}

$$
\eta=A \cdot e^{-\frac{E_{a}}{R \cdot T}}
$$

Donde: $\eta=\operatorname{viscosidad}(P a \cdot s)$

$A=$ constante de Arrhenius

$E_{a}=$ Energía de activación $(\mathrm{J} / \mathrm{mol})$

$T=$ Temperatura $\left({ }^{\circ} \mathrm{K}\right)$

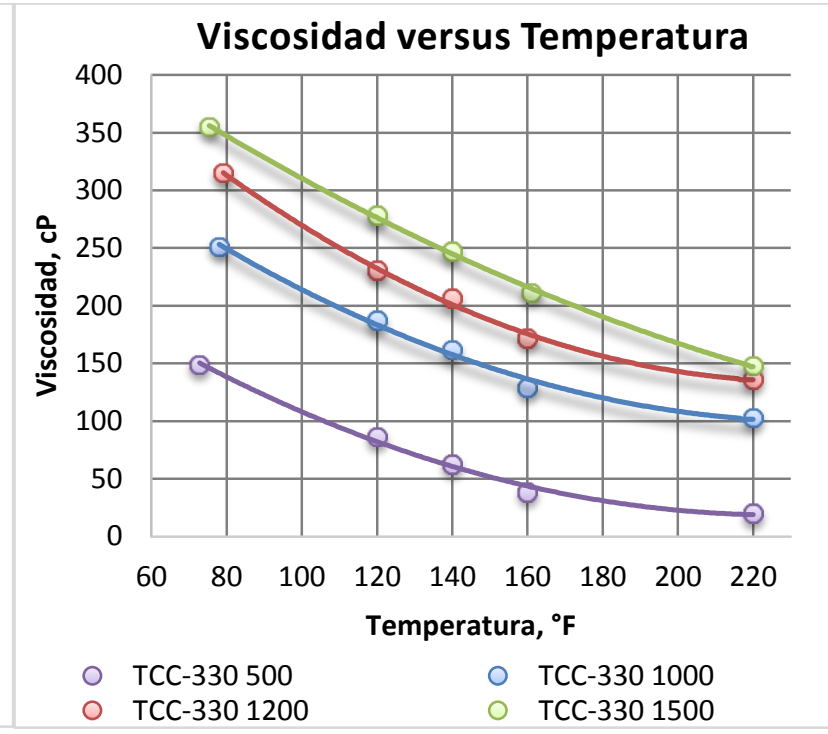

Figura 4. Resultados de Viscosidad de la solución de agua versus Concentración extrapolado a $220^{\circ} \mathrm{F}$ - TCC330

Ec. 1: Ecuación de Arrhenius 
Aplicando logaritmo natural a la ecuación 1 se tiene:

$$
\ln (\eta)=-\frac{E_{a}}{R}\left(\frac{1}{T}\right)+\ln (A)
$$

Ec. 2: Ecuación de Arrhenius linealizada

\subsection{Pruebas de Compatibilidad}

En esta prueba se evalúa el porcentaje de agua presente. Este porcentaje se valoró mediante la compatibilidad que tuvieron las soluciones de polímeros con el crudo (Lake, 1989). La compatibilidad se evalúo bajo criterios de observación de separación de fases y formación de emulsión. El polímero EOR909 no presentó una buena separación de las fases, por lo cual no se pudo apreciar una interface definida indicando la formación de emulsión. Por el contrario, el polímero TCC-330 mostró ausencia de emulsión y formación de una interface bien definida. En la mayoría de los resultados registrados a la temperatura de 160F y con una agitación baja se evidenció una separación de fases de manera rápida. Lo contrario se registró a temperaturas menores. Además, la visualización de las fases es difícil apreciar en concentraciones de menor grado y alta agitación.

En la Figura 5 se observa que el polímero TCC-330 en la concentración de 1.500 ppm tiene mejor compatibilidad con el crudo y un porcentaje de agua mayor.

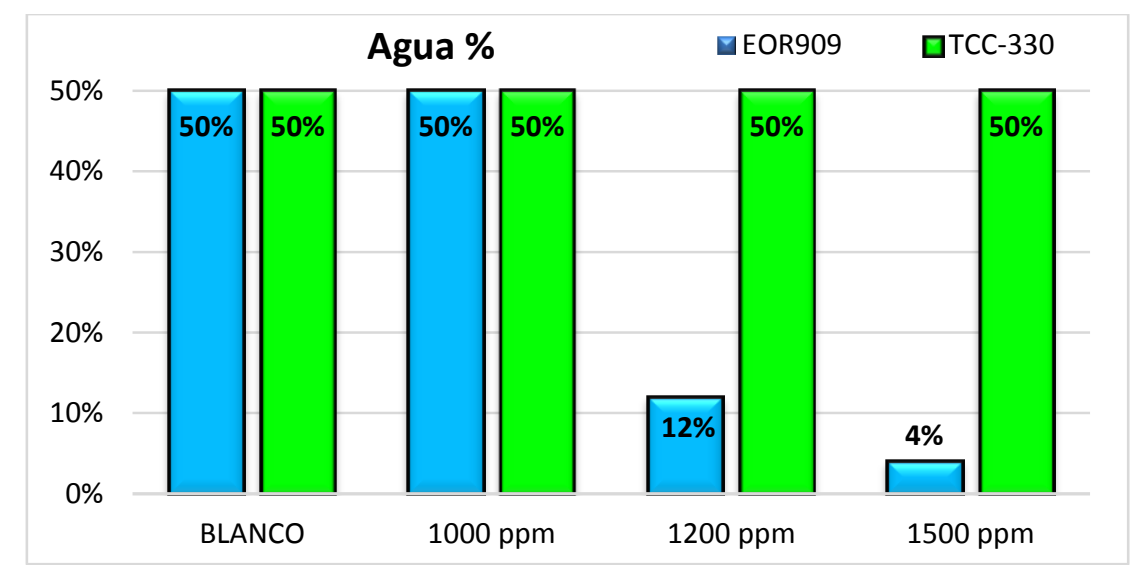

Figura 5. Resultados de pruebas de compatibilidad con crudo

\subsection{Pruebas de Adsorción}

Este ensayo es un indicativo cuantitativo de la cantidad de polímero que puede quedar adsorbida por la roca durante la inyección. En la Figura 6 se observa que al inundar con polímero a los cortes hay una reducción mínima de peso que corresponde a pérdidas durante el proceso de filtrado. En la Figura 7 se muestra que la impregnación de los polímeros usados en el ensayo es despreciable con valores menores al $1 \%$. Esto indica que tienen una baja probabilidad de adsorción. 


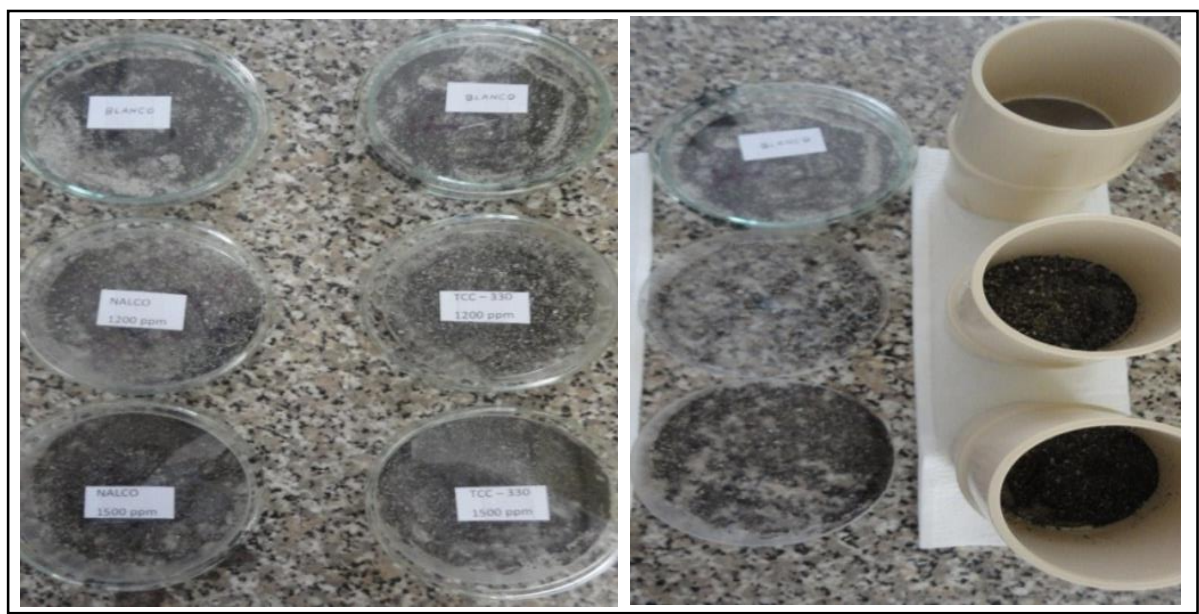

Figura 6. Ensayo de impregnación de polímero (lado izquierdo: solución polimérica con cortes, lado derecho: cortes filtrados)

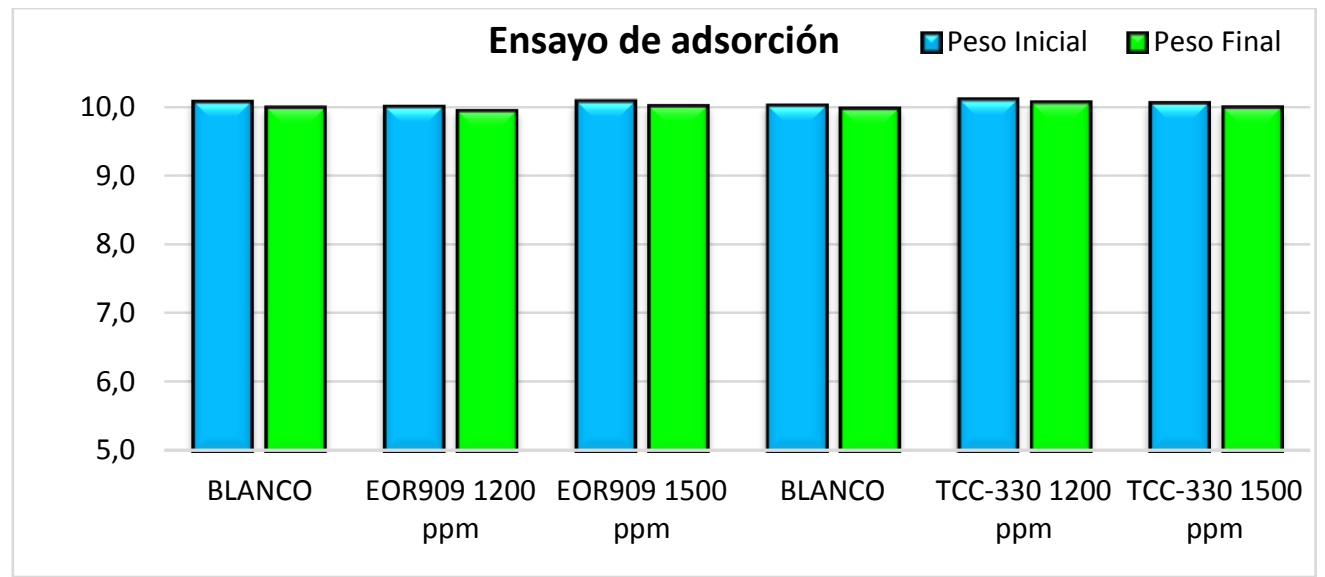

Figura 7. Peso de muestras antes y después de ensayo de adsorción

\subsection{Ensayo de Salinidad}

La Figura 8 muestra que la viscosidad se reduce aproximadamente a $80 \%$ al añadir $2 \%$ de $\mathrm{NaCl}$ en la solución de polímero TCC-330 y un 85\% al añadir 10\% de $\mathrm{NaCl}$. En cambio, en la solución de polímero EOR909 al añadir $2 \%$ de $\mathrm{NaCl}$ decrece abruptamente la viscosidad a un $90 \%$, y al añadir el $10 \%$ no se pudo medir la viscosidad a la solución debido que fue severamente paralizada la solución por la sal. Además, hace más ácida a las soluciones elevando la cantidad de cloruros presentes. Se pude concluir que la salinidad es un factor muy importante en la preparación de las soluciones poliméricas pues la viscosidad se reduce considerablemente en los dos ensayos.

\subsection{Pruebas de Desplazamiento}

En esta prueba se simula el desplazamiento del crudo al inyectar la solución de polímero a las diferentes concentraciones de 1.000 a 1.500 ppm. Para este ensayo se utilizó un equipo de desplazamiento lineal. Para ello se construyó un aparato donde se pueda hacer la inyección de los fluidos (Figura 9) y donde se pueda evaluar visualmente los resultados. 


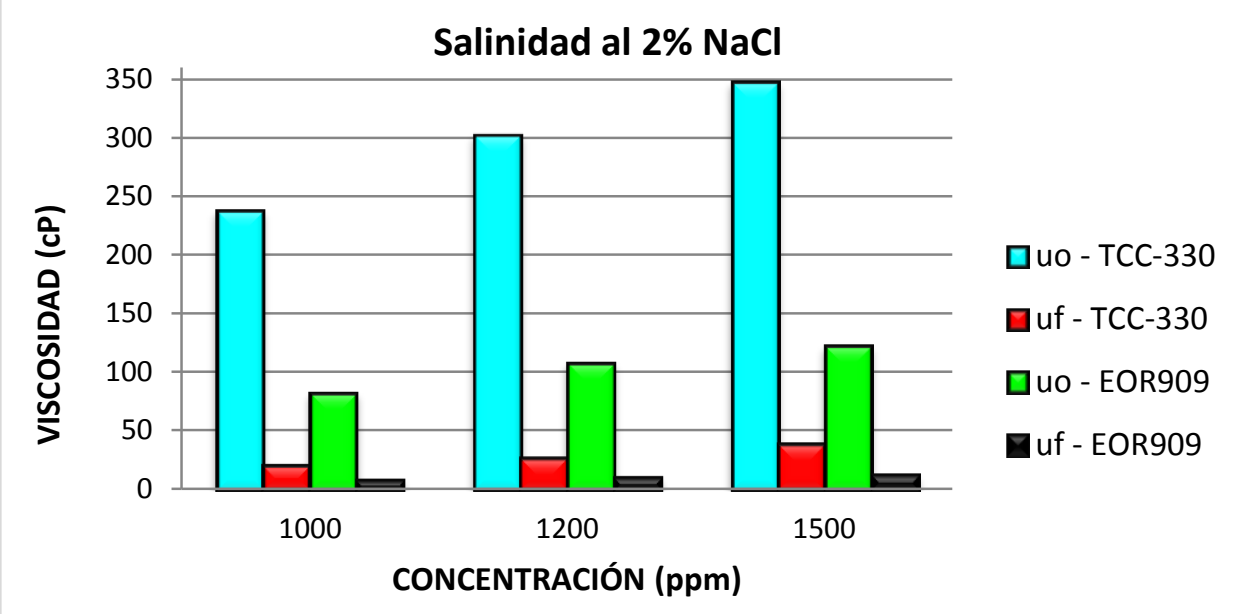

Figura 8. Viscosidad de las soluciones de polímeros al añadir $2 \% \mathrm{NaCl}$

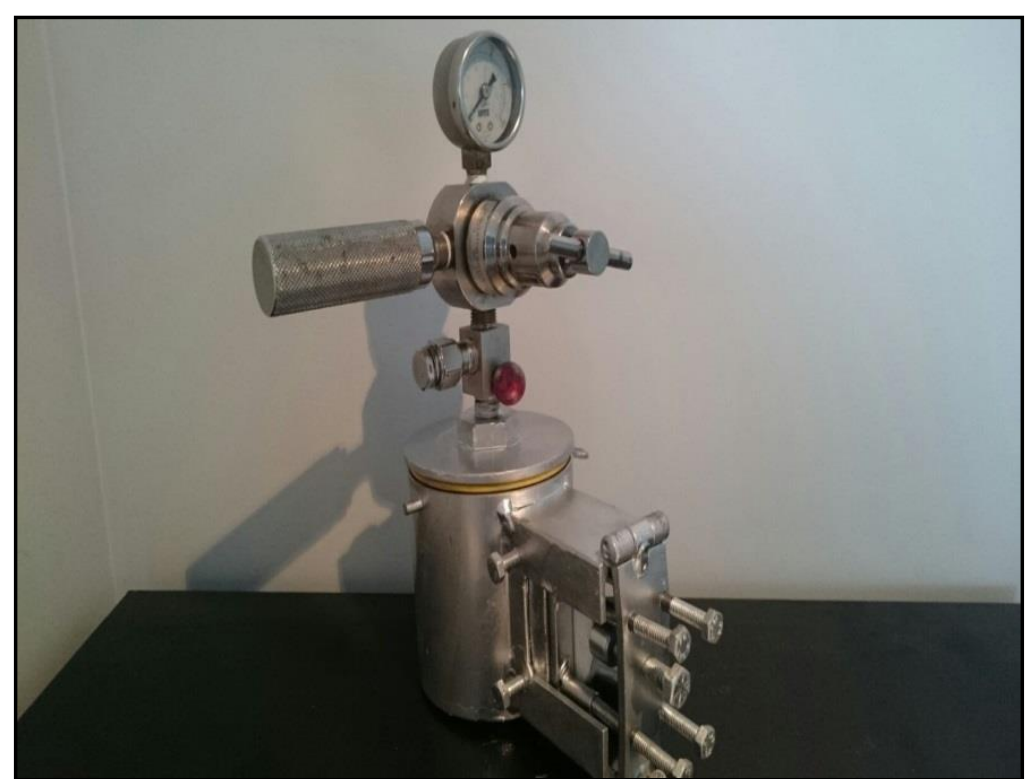

Figura 9. Equipo de desplazamiento diseñado para medir el desplazamiento de polímeros

\section{a) Descripción del equipo}

El equipo fue especialmente diseñado para el desarrollo de pruebas. Fue fabricado en acero inoxidable para evitar la rápida corrosión debido a su contacto con el hidrocarburo. Tiene un acople para colocar un manómetro que ayuda a inyectar presión (máximo 50 psi) y en el cuerpo de equipo un acople para adaptar los núcleos de cerámica como se ilustra en la Figura 9.

\section{b) Resultados}

En esta prueba se inyectaron soluciones de polímeros con concentraciones de 1.000 a 1.500 ppm. Para cada inyección se utilizó un núcleo nuevo. Esta prueba tiene carácter visual debido a que no cumple con todas las especificaciones para ser tomados como datos puntuales. Se evaluó de manera visual y matemática mediante cálculo del área de desplazamiento del crudo. El núcleo tiene un punto de referencia que se aprecia para poder observar y calcular el desplazamiento que ha ocurrido con la inyección de los polímeros (Figura 10). 


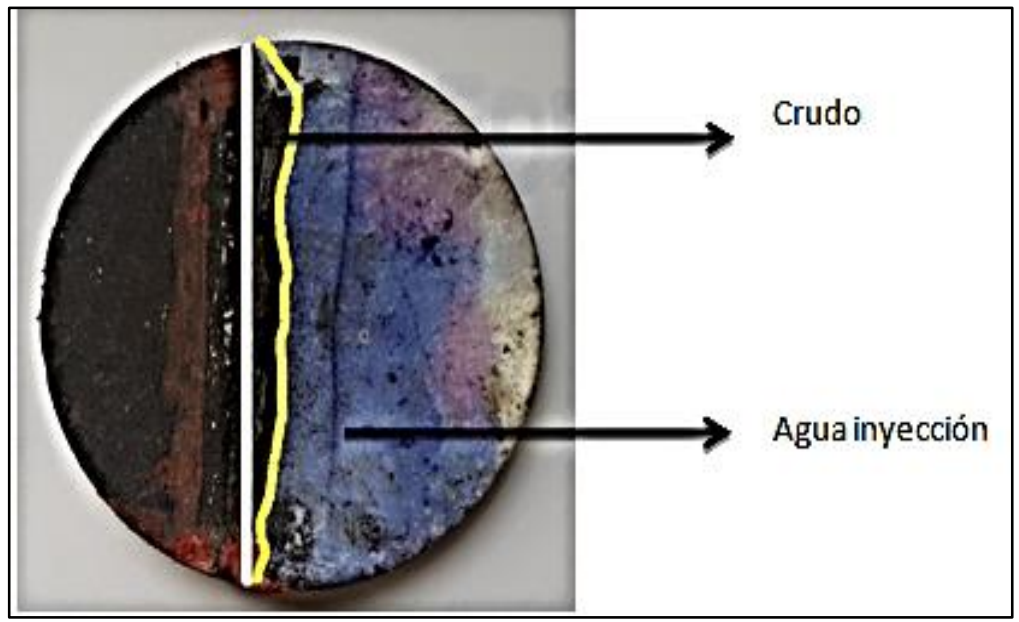

Figura 10. Núcleo inundado con polímero a 1.000 ppm

De la Figura 11 se puede deducir que el polímero TCC-330 tiene una eficiencia mayor para desplazar el crudo con respecto al polímero EOR909. También se observa que a mayor concentración el desplazamiento fue mayor e igual que el tiempo que este se demora.

Cabe mencionar que la concentración de polímero para el desplazamiento de crudo tiene un punto óptimo que debe ser tomado en cuenta y que está estrechamente ligado con las características del yacimiento. Esta prueba se realizó para evaluar el polímero que mejor relación de desplazamiento origina en el crudo y los porcentajes están dados bajo criterios de observación.

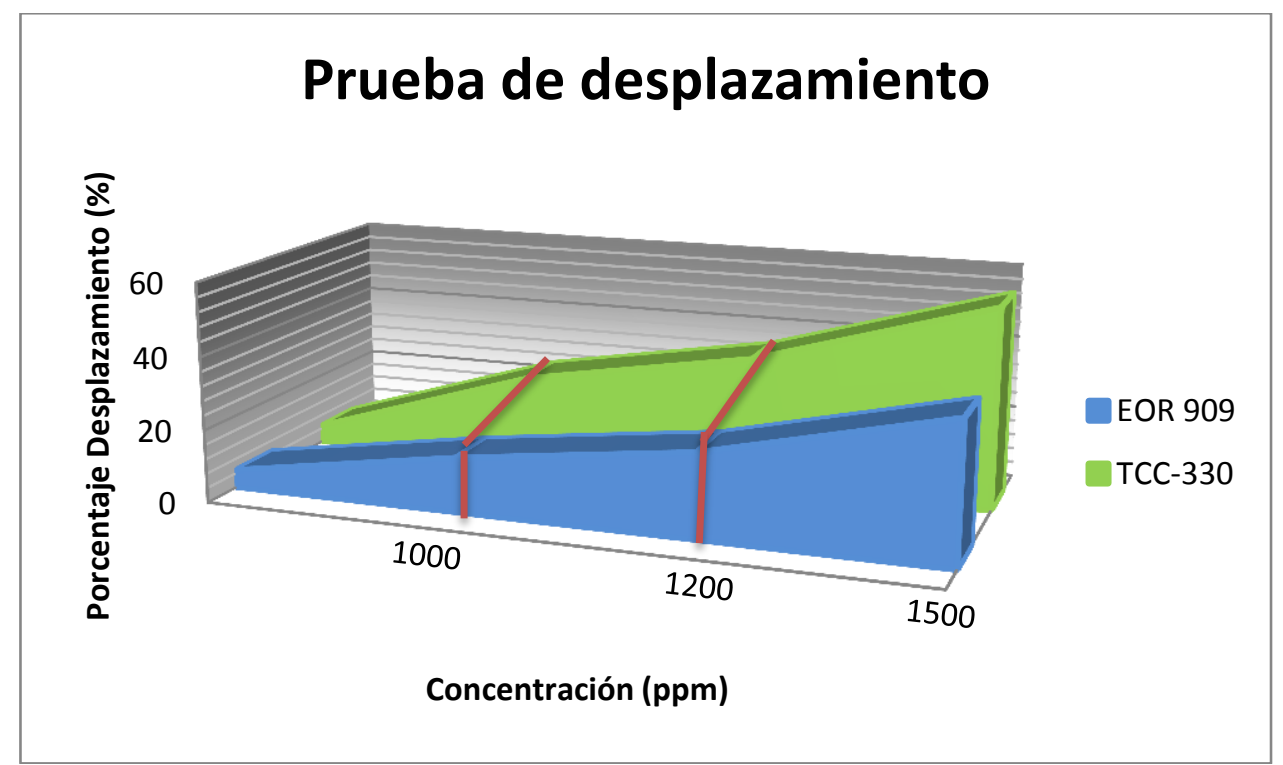

Figura 11. Desplazamiento con polímero EOR909 y TCC-330 a 1.500 ppm

\section{Conclusiones}

La solución de polímero TCC-330 a una concentración de 1.500 ppm fue la mejor formulación para la inyección en el yacimiento "X". A esta concentración de la solución se modificó la viscosidad del fluido desplazante (agua de inyección) al mezclarlo con polímero, reduciendo su movilidad, y mejorando la movilidad del crudo. Para alcanzar esta concentración óptima, se realizaron previamente mezclas a diferentes concentraciones: 500, 1.000, 1.200, 1.500 partes por 
millón. De estas, el que mejor resultado dio fue el de 1.500 ppm basado en el desplazamiento que se obtuvo en las muestras de cerámica.

Con base a la Tabla 2, el polímero TCC-330 presenta las mejores características físico-químicas; al ser un polímero de tipo poliacrilamida parcialmente hidrolizada, su grado hidrólisis es del $26 \%$, lo cual indica que es altamente activo. Sufre una degradación por calor inferior al $25 \%$, como muestra la Tabla 3, lo cual nos indica que es poco susceptible a los cambios por temperatura; soportando temperaturas mayores a $160^{\circ} \mathrm{F}$. Tiene un alto peso molecular, con la cual se obtuvieron viscosidades altas a distintas temperaturas. Es un polímero con carga aniónica que evita la interacción con los fluidos del reservorio y la roca yacimiento.

El polímero TCC-330 alcanza una viscosidad más alta con 1.500 ppm de concentración respecto al EOR909 que necesita una mayor concentración de 2.200 ppm para alcanzar la misma viscosidad, esto es debido al alto peso molecular de TCC-330.

Se evaluó mediante las figuras 3 y 4 que la viscosidad en función a la temperatura es una relación inversamente proporcional y que el polímero TCC-330 es susceptible en un 25\% a disminuir su viscosidad conforme se aumenta la temperatura. En cambio, el polímero EOR909 es más susceptible debido a que su viscosidad decrece en un $65 \%$ al incrementar temperatura. También se pudo apreciar que, al haber mayor temperatura, la viscosidad decrece en mayor escala en soluciones con menor concentración de polímero lo cual indica que el polímero TCC-330 es más estable al efecto térmico.

La compatibilidad se evalúo bajo criterios de observación de separación de fases y formación de emulsión. Como se detalla en la Figura 5, el polímero EOR909 no presentó una buena separación de las fases, por lo cual no se pudo apreciar una interfaz definida indicando la formación de emulsión. Por el contrario, el polímero TCC-330 mostró ausencia de emulsión y formación de una interfaz bien definida.

Los ensayos de salinidad mostraron que la viscosidad se reduce del $80 \%$ al $85 \%$ al añadir el $2 \%$ y el $10 \%$ respectivamente de $\mathrm{NaCl}$ en la solución de polímero TCC-330. En cambio, la solución de polímero EOR909, al añadir un $2 \%$ de sal, decreció abruptamente en un $90 \%$ su viscosidad y al añadir el $10 \%$ no se pudo medir el valor pues la solución fue saturada por la sal.

Las pruebas de adsorción muestran una impregnación despreciable de las soluciones de ambos polímeros menor al 1\%. Esto indica que pueden ser usados y con una baja probabilidad de adsorción y/o retención.

La Figura 11 corresponde a una ilustración estadística que muestra que el polímero TCC-330 a 1.500 ppm tiene el mayor desplazamiento con un porcentaje superior al 50\%, en comparación con 
un $38 \%$ de crudo desplazado con el polímero EOR909. Además, se observó que a mayor concentración hay mayor desplazamiento de crudo en el núcleo.

\section{Bibliografía}

Abrahamsen, A. (2012). Appling chemical EOR on the norne field C-segment. Tesis Maestría no publicada, Nowegian University of Science and Technology, Trondheim.

Aktas, F., Clemens, L., Castanier, L., \& Kovscek, A. (2008). Viscous oil displacement with aqueous associative polymers. SPE 113264, 2-5.

API. (1990). NORMA API RP-63 "Recommended Practices for Evaluation of Polymers used in Enhanced Oil Recovery Operations". Washington.

Barnes, A. L. (1962, octubre). The use of a Viscous Shug to impove Waterflood Efficiency. SPE 334-PA, 14(10), 1147-1153.

Buchgraber, M. (2008). Determination of the reservoir behaviour of associative polymers at various polymer concentrations. Tesis de Maestría, Universidad de Leoben, Austria.

Carcoana, A. (1992). Applied enhanced oil recovery. Prentice Hall, New Jersey.

Chinenye, C. (2010). Enhanced Oil Recovery for norme field's segment using surfactant flooding. Tesis de Maestría, NTNU Norwegian University of Science and Technology, Departamento de Ingeniería de Petróleos y aplicaciuones geofísicas, Spring.

Cuenca, V., \& Cóndor, J. (2016). Diseño experimental de una solución de polímero para mejorar la relación de movilidad en el yacimiento $x$ (Tesis). Quito: UCE-FIGEMPA. Retrieved from www.dspace.uce.edu.ec

Demin, W., Zhang, Z., Chun, L., Cheg, J., Du, X., \& Li, Q. (28-31 de 10 de 1996). A pilot for Polymer flooding of saertu formation S II in the North of Daqing Oil Field. En S. A. 37009 (Ed.), Pacific Oil and Gas Conference, (pág. 11). Adelaide - Australia.

Lake, L. W. (1989). Enhanced Oil Recovery (Vol. 35). New Jersey, USA: Pretencie - Hall.

Pancharoen, M. (2009). Physical Properties of AssociativePolymer Solutions. Master of Science, Stanford University, Departamnet of Energy Resources Engineering, Oklahoma.

Qmax Solutions S.A. (2015). Informe Hojas Técnicas de Polímeros 2015. Informe de parámetros técnicos de polimeros 2015 para EOR, Laboratorio Qmax Solutions S.A., Quito.

Salager, J.-L. (2005). Recuperación Mejorada del Petróleo. Mérida-Venezuela: Universidad de los Andes.

Sánchez Barrios, L. (2013, marzo 31). tecnicasderecuperacionmejorada.blogspot.com. Retrieved diciembre 06, 2015, from http://tecnicasderecuperacionmejorada.blogspot.com/ 
Stosur, G. J., Hite, J. R., Carnahan, N. F., \& Miller, K. (2003). The Alphabet Soup of IOR, EOR and AOR: Effective Communication Requires a Definition of Terms. SPE paper 84908, 1-3.

Xu, H., Kim, D. H., \& Delshad, M. (2012, diciembre). Potential for Non-thermal Cost-effective Chemical Augmented Waterflood for Producing Viscous Oils. SPE -165350-MS, 1-3. 